\title{
Training Dual Tasks Together or Apart in Parkinson's Disease: Results From the DUALITY Trial
}

\author{
Carolien Strouwen, PhD, ${ }^{1}$ Esther A. L. M. Molenaar, MSc, ${ }^{2}$ Liesbeth Münks, MSc, ${ }^{1}$ Samyra H. J. Keus, PhD, ${ }^{2}$ \\ Jan C. M. Zijlmans, MD, ${ }^{3}$ Wim Vandenberghe, MD, ${ }^{4,5}$ Bastiaan R. Bloem, MD, ${ }^{6}$ and Alice Nieuwboer, PhD $^{1 *}$ \\ ${ }^{1}$ Department of Rehabilitation Sciences, Faculty of Kinesiology and Rehabilitation, KU Leuven, Leuven, Belgium \\ ${ }^{2}$ Department of Neurology, Nijmegen Centre for Evidence Based Practice, Radboud University Medical Centre, Nijmegen, The Netherlands \\ ${ }^{3}$ Department of Neurology, Amphia Hospital, Breda, The Netherlands \\ ${ }^{4}$ Department of Neurology, University Hospitals Leuven, Leuven, Belgium \\ ${ }^{5}$ Department of Neurosciences, KU Leuven, Leuven, Belgium \\ ${ }^{6}$ Department of Neurology, Donders Institute for Brain, Cognition and Behaviour, Radboud University Medical Centre, Nijmegen, The Netherlands
}

\begin{abstract}
Background and Objectives: Many controversies surround the usefulness of dual-task training in Parkinson's disease (PD). This study (1) compared the efficacy of two different dual-task training programs for improving dual-task gait and (2) assessed the possible fall risk of such training.

Methods: Patients $(N=121)$ with a diagnosis of PD (aged $65.93[ \pm 9.22]$ years, Hoehn and Yahr stage II-III on-medication) were randomized to (1) a consecutive group in which gait and cognitive tasks were trained separately or (2) an integrated group in which gait and cognitive tasks were trained simultaneously. Both interventions involved 6 weeks of at-home physiotherapistled training. Two baseline tests were performed as a 6week control period before training. Posttests were performed immediately after training and at 12-week follow-up. Dual-task gait was assessed during trained and untrained secondary tasks to assess consolidation of learning. Fall risk was determined by a weekly telephone call for 24 weeks.
\end{abstract}

Results: No significant time by group interactions were found, suggesting that both training modes had a similar effect on dual-task gait. Immediately after training, and not after the control period, significant improvements $(P<.001)$ in dual-task gait velocity were found in all trained and untrained dual tasks. Improvements ranged between $7.75 \%$ and $13.44 \%$ when compared with baseline values and were retained at 12-week follow-up. No significant change in fall risk occurred in both study arms $(P=.84)$.

Conclusions: Consecutive and integrated dual-task training led to similar and sustained improvements in dual-task gait velocity without increasing fall risk. These novel findings support adoption of dual-task training in clinical practice. () 2017 International Parkinson and Movement Disorder Society

Key Words: executive function; falls; gait; rehabilitation; Parkinson's disease
${ }^{*}$ Corresponding author: Prof. Dr. Alice Nieuwboer, Department of Rehabilitation Sciences, KU Leuven, Tervuursevest 101/1501, B-3000 Leuven, Belgium; Alice.Nieuwboer@kuleuven.be

Funding agencies: Financial support for the study was provided by the Jacques and Gloria Gossweiler Foundation and by the Malou Malou funds of the King Baudouin Foundation.

Relevant conflicts of interests/financial disclosures: The funding sources were not involved in study design or execution. All researchers involved in the study were independent from the funders.

Trial Registration: Clinicaltrials.gov NCT01375413.

Received: 6 October 2016; Revised: 13 March 2017; Accepted: 17 March 2017

Published online 00 Month 2017 in Wiley Online Library (wileyonlinelibrary.com). DOI: 10.1002/mds.27014
Parkinson's disease (PD) is characterized by loss of automatic motor control ${ }^{1}$ and cognitive impairment, most notably by executive dysfunction. ${ }^{2-4}$ Dual-task (DT) performance relies on the capacity to perform motor tasks automatically as well as on the cognitive (executive) ability to integrate different task demands. Several studies demonstrated compromised DT gait in mid to moderate stages of $\mathrm{PD}, 5,6$ correlating with an increased risk of falling, ${ }^{7}$ more freezing of gait (FOG) ${ }^{8}$ and reduced functional mobility. ${ }^{9}$

Physiotherapy guidelines state that DT training is better avoided $^{10}$ or at best undertaken cautiously. ${ }^{11}$ However, a recent review of uncontrolled pilot studies $^{12}$ together with 1 randomized controlled trial ${ }^{13}$ 
showed benefits of DT training in PD. So far, no motor-cognitive training studies have been conducted with a priori determined sample sizes for optimal effect-size estimation to ascertain the difference in benefits of 2 different DT training programs administered in a home setting. ${ }^{12}$ What is, unknown is how DT training is best performed and whether it should focus on automatization of each task separately or on task integration. $^{5,12,14}$ Therefore, we designed the DUALITY study to investigate the effects of 2 DT training programs and their possible fall risk delivered in the home setting. Integrated dual-task training (IDT) consisted of simultaneous gait practice and cognitive exercises to optimize DT integration. In contrast, consecutive task training (CTT) delivered gait and cognitive exercises separately to improve task automaticity. ${ }^{15}$ In PD, the transfer of learning is compromised. This means that the generalization of learning to skills that were not incorporated in the training program is reduced. Therefore, we hypothesized that IDT would have larger effects than CTT because of its greater inherent cognitive challenge, resulting in better retention and transfer to daily life. ${ }^{14}$ On the downside, IDT was expected to enhance fall risk as attention would be diverted from gait. ${ }^{10}$ Finally, we explored which clinical characteristics influenced the effect of training.

\section{Methods}

\section{Study Design and Participants}

The study involved a multicentred, single-blind, randomized clinical trial. The University Hospitals Leuven (Belgium) and the Radboud University Medical Center Nijmegen (the Netherlands) received ethical approval for the study (Belgium: Commissie Medische Ethiek (CME) University of Leuven (KU) Leuven B322201213165/S53419 and the Netherlands: Commissie Mens-gebonden Onderzoek (CMO) Regio Arnhem-Nijmegen - NL39530.091.12). The DUALITY trial was registered in clinicaltrials.gov as NTC01375413. In a detailed description of the protocol and power calculation, we revealed that a sample of 120 participants would be needed to detect a clinically meaningful difference of $15 \%$ between IDT and CTT for the primary outcome DT gait velocity. ${ }^{15}$

Inclusion criteria were (1) diagnosis of PD according to the UK Brain Bank criteria, ${ }^{16}$ (2) Hoehn and Yahr (H\&Y) stages II-III when on medication, ${ }^{17}$ (3) able to walk 10 minutes continuously, (4) presence of DT interference established by a structured checklist, ${ }^{15}$ (5) Mini Mental State Examination $\geq 24,{ }^{18}$ (6) stable medication over the past 3 months, (7) no hearing or visual problems interfering with testing or training, and (8) no deep brain stimulator or stable deep brain stimulator settings during the past year. Participants were excluded when they presented with unstable other medical conditions that affected gait. Other physiotherapy interventions were continued during the trial and their content was documented, but we specifically asked therapists not to address dual tasking, gait and cognitive exercises.

Participants signed an informed consent form before participation. Measurement protocols and treatment interventions were standardized across both centers, and fidelity checks were carried out through site visits. Fall frequency and adverse effects were monitored by a review board to ensure participants' safety. Testing was performed by a blinded physiotherapist at 4 time points while participants were on medication. After baseline measurement (test 1) and a 6-week interval, a second baseline measurement was performed (test 2) to serve as a control period. This allowed monitoring of fall frequency prospectively before intervention. A third test was carried out immediately after the 6week training (test 3). A follow-up test was performed after 12 weeks without training (test 4).

\section{Intervention}

Both interventions were delivered at home by a physiotherapist from each center specifically trained for the aims of the study. Supervised training frequency was twice a week for 6 weeks. Practice time was equal in both interventions and consisted of 40 minutes of active exercise per session. In addition, the participants were asked to perform unsupervised exercises twice a week for 30 minutes. In the CTT arm, the participants performed 15 minutes of gait practice, 15 minutes of cognitive exercises in sitting, and 10 minutes of functional practice based on breaking down tasks in separate components. A detailed description of training contents was published earlier. ${ }^{15}$ Cognitive exercises involved verbal fluency tasks and reciting switching and working memory tasks. ${ }^{15}$ IDT consisted of 30 minutes of concurrent practice of gait and the same cognitive exercises as in CTT. The 10 minutes of functional training included integrated DT practice. Training diaries were checked by the therapists to monitor compliance. In both groups, cognitive exercises were offered at different difficulty levels ${ }^{15}$ to provide sufficient challenge for each participant and to ensure a structured training program in both centers. Progression to the next level was determined by flawless performance at the chosen training tasks and was scored accordingly by the treating physiotherapist $(+1=$ progression, $0=$ same level) allowing to sum the progression scores of each patient.

\section{Randomization and Masking}

After screening for eligibility, participants were randomly assigned to the IDT or CTT training groups in 
TABLE 1. Descriptive characteristics at baseline test 1

\begin{tabular}{|c|c|c|c|c|}
\hline & Total group, $\mathrm{N}=121$ & $\mathrm{CT}, \mathrm{n}=65$ & IDT, $\mathrm{n}=56$ & $\begin{array}{c}\mathrm{CTT} \text { versus IDT, } \\
P \text { value }\end{array}$ \\
\hline Age,y & $65.93 \pm 9.22$ & $66.05 \pm 9.30$ & $65.80 \pm 9.19$ & .89 \\
\hline Gender, M/F & $88 / 33$ & $49 / 16$ & $39 / 17$ & .48 \\
\hline Disease duration, y & $8.67 \pm 5.83$ & $8.89 \pm 6.30$ & $8.41 \pm 5.29$ & .65 \\
\hline Freezing of gait, yes/no & $68 / 53$ & $34 / 31$ & $34 / 22$ & .35 \\
\hline $\begin{array}{l}\text { Recurrent falls } 6 \text { months } \\
\text { prior to study, yes/no }\end{array}$ & $40 / 81$ & $22 / 43$ & $18 / 38$ & .84 \\
\hline LED, mg/day & $687.80 \pm 431.66$ & $752.25 \pm 453.24$ & $612.99 \pm 396.10$ & .08 \\
\hline DBS, yes/no & $18 / 103$ & $9 / 56$ & $9 / 47$ & .73 \\
\hline MMSE, /30 & $27.94 \pm 1.59$ & $27.88 \pm 1.65$ & $28.02 \pm 1.53$ & .63 \\
\hline MoCA, /30 & $25.81 \pm 2.78$ & $25.71 \pm 2.82$ & $25.93 \pm 2.76$ & .67 \\
\hline $\mathrm{FAB}, / 18$ & $15.76 \pm 1.99$ & $15.77 \pm 1.97$ & $15.75 \pm 2.03$ & .96 \\
\hline$H \& Y, \| / I I I$ & $80 / 40$ & $42 / 23$ & $38 / 17$ & .60 \\
\hline
\end{tabular}

Means and standard deviations are presented. CTT, consecutive task training; IDT, integrated task training; N, number of participants; LED, levodopa equivalent dose; DBS, Deep Brain Stimulator; MMSE, Mini Mental State Examination; MoCA, Montreal Cognitive Assessment; FAB, Frontal Assessment Battery; H\&Y, Hoehn \& Yahr stage; H\&Y stage was not recorded for 1 participant.

each center. A computerized block randomization procedure (block size $=4$ ) was implemented by an independent statistician. Group allocation was performed by an independent person, who contacted the treating physiotherapist by email to ensure concealed allocation. Randomization was stratified by subgroup with or without FOG and by stage of the disease (H\&Y stages II and III).

\section{Outcome Measures}

The primary outcome was gait velocity during the auditory Stroop task demonstrated to have excellent test-retest reliability. ${ }^{19}$ This task was not trained and therefore tested the transfer of learning. Secondary outcomes were gait velocity while performing a backward digit span task (trained) and while using a mobile phone (untrained) to assess transfer to daily life. Details on the additional tasks can be found in Supplementary Table 1.

Gait was recorded via the GAITRite Walkway system $^{20}$ with an active length of $7.92 \mathrm{~m}$ and a sampling frequency of $120 \mathrm{~Hz}$. During DT conditions, participants were asked to "walk over the walkway at preferred speed while performing the secondary task and divide attention equally across both tasks." All tasks were additionally performed under single-task (ST) conditions. ST and DT test order was randomized but kept constant for each patient throughout the trial. Cognitive task performance was synchronized with walking and verbal responses were recorded via a microphone mounted on a headphone set (Supplementary Table 1).

Falls were evaluated for 24 weeks by weekly phone calls by a blinded tester while referring to the following definition of falling: "coming to rest unintentionally on the ground or other lower level, when this occurs not as the result of a major intrinsic event or overwhelming hazard." ${ }^{21}$ Fall frequency was calculated for four 6-week periods: (1) between the 2 baseline tests, (2) during the intervention, (3) during the 6-week period immediately following the intervention, and (4) during the final 6 weeks of follow-up. At trial exit, patients' perceptions of the training were assessed by a structured interview.

\section{Statistical Analysis}

Statistical analysis was performed according to the intention-to-treat principle, including all participants initially included in the study (IBM SPSS version 22; IBM Corp., Armonk, New York). ${ }^{22}$ Missing data $(2 \%-13 \%)$ were the result of dropout $(6.61 \%)$, technical problems, and human errors. Linear mixed models (using a direct likelihood estimation technique) were used to analyze all continuous outcomes as values were missing at random. Two-sided $P$ values were set at $\alpha$ level 0.05 . Treatment group (IDT vs CTT), time (test 1-4) and the interaction between time and group were used as fixed factors. Participants were included as random factor. We calculated medication dose as levodopa equivalent dose (LED) ${ }^{23}$ and explored its confounding effect. Fall frequency data was analyzed using a negative binomial model. A separate analysis compared whether people who dropped out of the study differed from those who completed it. The influence of different subgroups, that is, with and without FOG, falls, H\&Y stage, and cognition, was explored by including each term and their interactions in the model in separate analyses.

\section{Results}

Table 1 presents the descriptive characteristics of the participants $(\mathrm{N}=121)$. As shown in Figure 1, 65 participants were randomized into CTT and 56 into IDT. Blinded testers guessed correctly in which group 


\section{Flow Diagram}

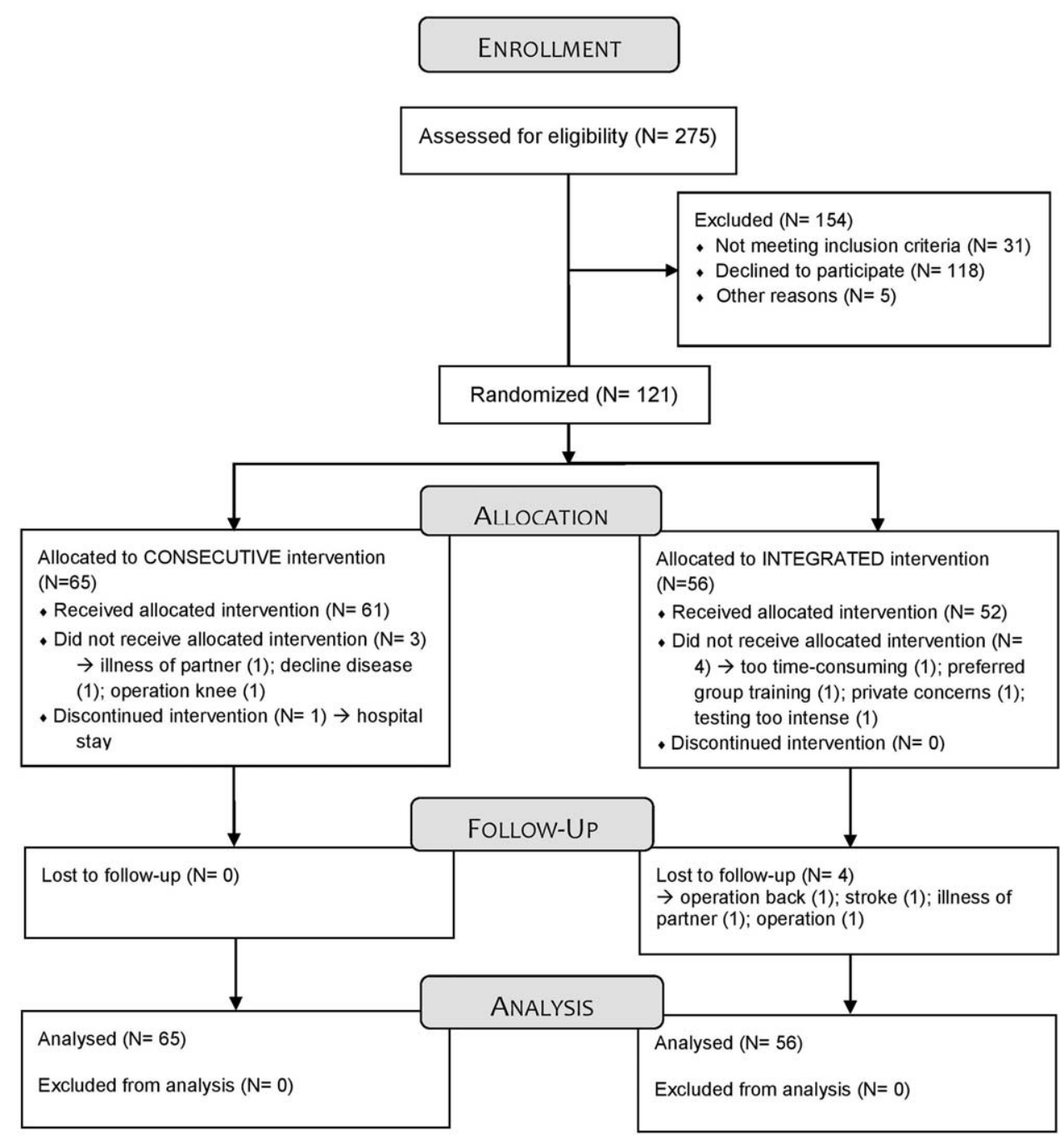

FIG. 1. Flow diagram of the DUALITY trial. N, number of participants.

participants were randomized in $58.2 \%$ of cases, illustrating that blinding was successful. Groups were similar for all disease characteristics, including LED (Table 1). However, Table 2 shows that LED was not stable in time $(F=7.41, P<.001)$ with higher doses at tests 3 and 4 . Therefore, LED was adopted as a covariate in all subsequent analyses.

Figure 1 illustrates that 4 participants dropped out of CTT. Of these participants, 3 did not receive CCT and 1 did not complete the intervention because of a hospital stay. A total of 8 participants dropped out of IDT. Of these participants, 4 did not receive IDT and 4 received the intervention but were lost to follow-up. Total dropout rate was $9.9 \%$. Reasons for dropout mostly involved health or time constraints, unrelated to the nature of the intervention. People who dropped out were not significantly different from those who completed the study.

Training diaries revealed that 6 participants in CTT and 6 in IDT followed less than $80 \%$ of the training sessions. Reasons for lack of compliance were lack of time, technical problems with the MP3 player used during home exercise, illness, and fatigue. In total, $84.6 \%$ of patients in CTT and $82.1 \%$ in IDT completed more than $80 \%$ of supervised and unsupervised training sessions. Analysis of progression levels of the cognitive exercises revealed that the CTT group advanced to significantly higher difficulty levels when compared with the IDT group (3.02 \pm 0.71 vs $2.71 \pm$ $0.81, \mathrm{~T}=2.14, P=.04)$. Fewer than half of the participants $(47 \%)$ continued their routine physiotherapy, including strengthening of lower and upper limb muscles, mobilization exercises, aquagym, physical fitness training, and various interventions for nonParkinson-related conditions.

\section{Primary and Secondary Dual-Task Outcomes}

In contrast to our hypotheses, no interaction effects between Time and Group were detected (Stroop: 


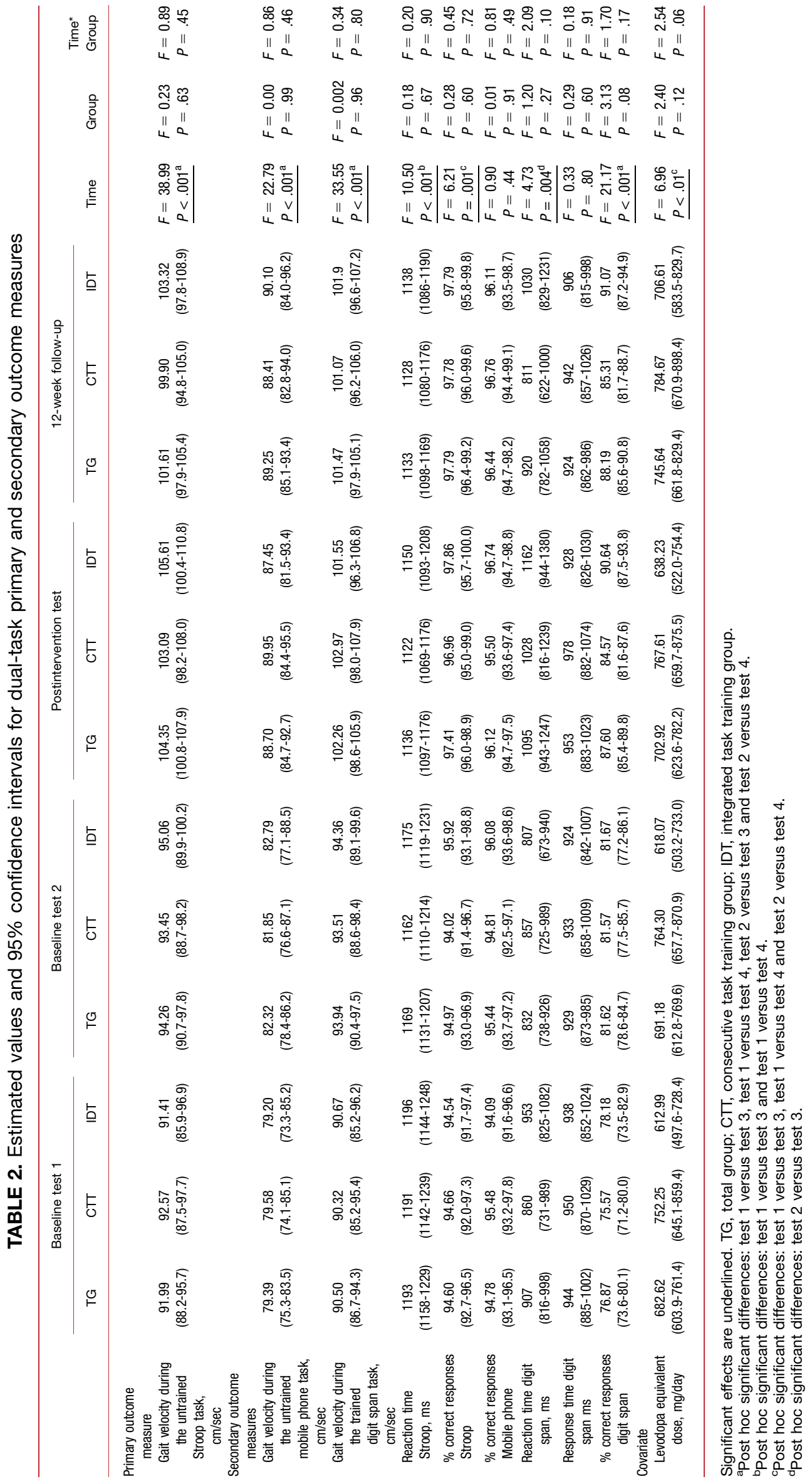




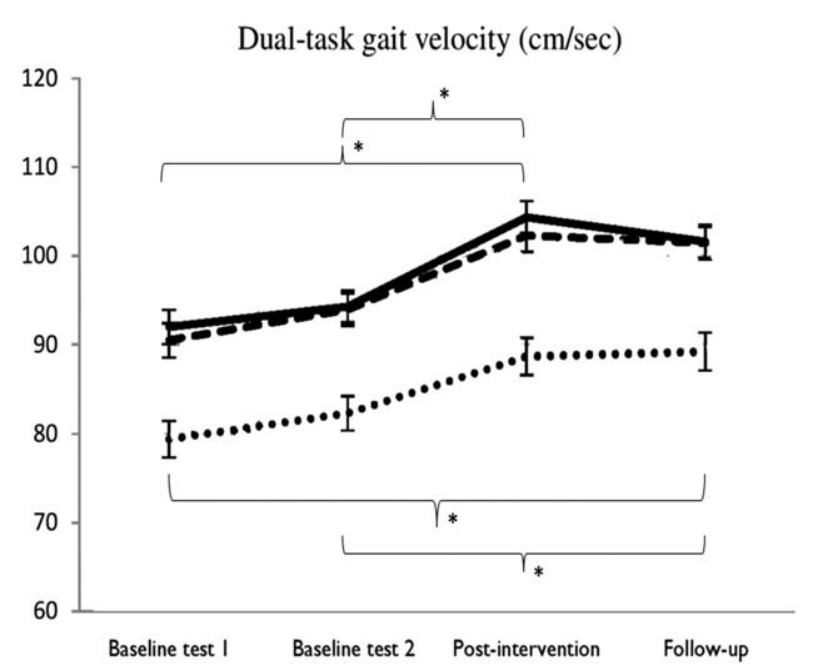

FIG. 2. Dual-task gait velocity $(\mathrm{cm} / \mathrm{sec})$ at each test session. The solid black line (_) represents gait velocity in combination with the untrained auditory stroop task (primary outcome), the dashed line (---) represents gait velocity during the trained backward digit span task, and the dotted line (...) represents gait velocity while performing the untrained mobile phone task. Error bars represent the standard errors of the mean. Significant contrasts of the linear mixed models are indicated by the black lines under the graph.

$F=0.89, P=.45$; mobile phone: $F=.86, P=.46$; digit span: $F=0.34, P=.80$; Table 2 ), indicating that both training strategies led to similar results.

Main effects for time were found for all 3 dual tasks (Stroop: $F=38.99, P<.001$; mobile phone: $F=$ $22.79, P<.001$; digit span: $F=33.55, P<.001$ ), indicating that both training strategies led to improved DT gait velocity when compared with the control period. As shown in Figure 2, the primary outcome (DT gait velocity with Stroop) was stable between baseline tests 1 and 2 and improved after the intervention $(\Delta$ with baseline 1: $P<.001$, effect size $[\mathrm{ES}]=$ $0.60 ; \Delta$ with baseline $2: P<.001$, ES $=0.53)$. These effects remained during follow-up (Table 2).

Similarly, the secondary outcomes, DT gait velocity with mobile phone (functional transfer) and with digit span (trained task), did not change significantly between baseline tests, improved after training and showed sustained benefits at follow-up (Fig. 2, Table 2). Subanalysis, excluding patients who dropped out or showed less than $80 \%$ compliance, revealed similar results compared to the intention-to-treat analysis. The results demonstrated that ST gait velocity followed the same pattern of improvement as DT gait velocity (Supplementary Table 2).

No interaction effects between time and group were found for DT cognitive outcomes (Table 2). Reaction time on the untrained DT Stroop remained stable between tests 1 and 2, improved after training when compared with baseline test $1(P=.001$, ES $=0.29)$, and remained better at follow-up. The percentage of correct responses on the DT Stroop showed the same pattern. In contrast, the percentage of correct responses on the DT mobile phone showed no improvements after training $(F=0.90, P=.44$; Table $2)$. Reaction time on the DT digit span remained stable between both baseline tests and worsened after training (when compared with baseline test 2). However, although the percentage of correct answers on this task was stable between both baseline tests, it improved significantly after training $(F=21.17, P<$ $.001)$, a result that was retained at follow-up. ST cognitive outcomes followed a similar pattern as DT cognitive outcomes (Supplementary Table 2).

\section{Fall Frequency}

No differences between both training groups were seen in the total number of fallers or in the total number of recurrent fallers (Table 3). Expressed as incidence rate ratios, both groups experienced $12 \%$ (95\% confidence interval $[\mathrm{CI}]=-60 \%$ to $94 \%$ ) fewer falls during the training period when compared with the preintervention period, $24 \%(95 \%$ CI $=-67 \%$ to $76 \%$ ) fewer falls in the 6 weeks right after the training period, and $6 \%(95 \% \mathrm{CI}=-54 \%$ to $142 \%)$ more falls in the final follow-up period (Table 3). These differences did not reach statistical significance $(P=$ .84). In the CTT group, 1 fall was recorded during unsupervised practice. No other adverse events were reported.

\section{Perception of Training During Exit Interviews}

Irrespective of training allocation, $89.9 \%$ of participants subjectively reported improvement during the training sessions. Of the participants, $85 \%$ perceived DT training as valuable for activities of daily life, and $75.2 \%$ reported using the exercises during activities of daily life, including the cognitive exercises. Interestingly, CTT found the training $\left(\chi^{2}=10.54, P=.001\right)$ significantly more useful during activities of daily life and stated they actually used it in daily life $\left(\chi^{2}=7.46\right.$, $P=.006)$. Of the participants, $75 \%$ had no problems working with the MP3 player, and $90 \%$ of the participants were prepared to continue the same training if it were offered again. A total of $77 \%$ of the participants felt that they were randomized to the best training group.

\section{Subgroups With Freezing, Falling, Higher H\&Y Stage, and Impaired Cognition}

No interaction effects of time by subgroup and type of training were found, indicating that patients with higher H\&Y stage $(n=40)$, with falls $(n=68)$, or with more cognitive impairment based on the Montreal Cognitive Assessment $<27(\mathrm{n}=65)$ benefited equally from both training modes. Secondary analyses for the primary outcome measure, including FOG as a fixed factor, revealed that people with FOG $(n=68)$ 
walked more slowly than people without $(94.1 \mathrm{~cm} / \mathrm{sec}$ [95\% CI $=89.9 \mathrm{~cm} / \mathrm{sec}$ to $98.4 \mathrm{~cm} / \mathrm{sec}$ ] vs $103.6 \mathrm{~cm} /$ $\sec [95 \% \mathrm{CI}=98.7 \mathrm{~cm} / \mathrm{sec}$ to $108.6 \mathrm{~cm} / \mathrm{sec}])$. Groups with and without FOG showed stable baseline measurements and improved DT performance after training. However, and in contrast to nonfreezers, freezers only showed retention in comparison to test 1 ( $\Delta$ with test $1=6.3 \mathrm{~cm} / \mathrm{sec}[95 \% \mathrm{CI}=1.0 \mathrm{~cm} / \mathrm{sec}$ to 11.5 $\mathrm{cm} / \mathrm{sec}], P=.01)$, but not when compared with test 2 $(\Delta=4.0 \mathrm{~cm} / \mathrm{sec}[95 \% \mathrm{CI}=-0.7 \mathrm{~cm} / \mathrm{sec}$ to $8.6 \mathrm{~cm} /$ sec], $P=.15)$.

\section{Discussion}

This study presents the first randomized clinical trial that investigated the effects of 2 DT training programs focusing on task integration on the one hand and separate task automatization on the other in a large cohort of early to mid-stage PD. The results showed that DT gait improved when compared with a control period without training, irrespective of which training method was used. Remarkably, effects transferred to untrained dual tasks and benefits were retained after 12-week follow-up, signifying consolidated learning effects. Furthermore, the novel training programs delivered in the community setting did not increase the risk of falls and showed excellent compliance rates.

In contrast to our hypothesis and earlier results in balance-impaired elderly, ${ }^{14}$ no differences between CTT and IDT were found. The observed DT performance improvements may thus be explained by better task automatization after CTT as well as by more efficient integration of task-related networks after IDT leading to the same result. Alternatively, the fact that both programs consisted of the same components may have contributed to this result, as ST performance improved for both gait and cognitive tasks, suggesting that task automatization may have played a large role in both groups. In this respect, our findings are contradictory to previous work ${ }^{24,25}$ showing that transfer of learning was decreased after CTT. Another reason for the lack of differences between CTT and IDT is that we individualized the training program for each participant. Although we equalized the amount of practice in both arms, the CTT group performed the cognitive exercises at a higher difficulty level than IDT, possibly because the former could fully concentrate on cognitive performance. This may explain why DT performance improved in CTT to the same degree as in IDT. Unfortunately, the exact training mechanisms cannot be gained from the current behavioral data and need to be addressed in future imaging studies of the underlying neural network changes associated with each training approach. 
Although not the main research question, we demonstrated DT training effects in the full cohort in contrast to a period without training of the same duration, controlling for repetition effects. However, because of the lack of an actual control group with either a placebo or no intervention, we cannot entirely exclude nonspecific effects as an explanation for these benefits. Despite this limitation, we showed that DT training transferred to untrained tasks such as the DT mobile phone and DT Stroop tasks, illustrating the functional relevance of the findings. The size of these improvements was modest, indicating a standardized effect size of 0.60 (Cohen's d) on the primary outcome with improvements ranging between $7.8 \%$ and $13.4 \%$ of baseline DT gait velocity. This improvement is comparable to what was found in previous studies, investigating the training of cognitive combined with (virtual) motor tasks in PD. ${ }^{12,26-28}$

Improvements exceeded the minimally clinically significant change for DT gait velocity determined for older adults, ${ }^{29}$ but not for PD. ${ }^{19}$ According to the former criterion, improvements during the digit span and Stroop were substantial $(>10 \mathrm{~cm} / \mathrm{sec})$ and small for the mobile phone task (between $5 \mathrm{~cm} / \mathrm{sec}$ and $10 \mathrm{~cm} /$ sec), which was also the more difficult task. ${ }^{19}$ This points to the possibility that more complex multitasks may lend themselves less well for transfer of training or alternatively that more multitasking should have been incorporated into the program. However, future work needs to determine first whether complex multitask training is at all feasible in PD.

In contrast to what we expected, transfer effects were present in both training groups in line with a previous cognitive-motor training study in PD. ${ }^{30}$ The similarities between the CTT and IDT programs may underlie this finding. It is also possible that, because we found only modest training effects $(E S=0.29$ ) for cognitive outcomes and strong effects for gait velocity $(\mathrm{ES}=0.60)$, the transfer of learning was predominantly driven by gait velocity increases.

Another interesting result was the significant improvement on reaction time and percentage of correct responses on the DT Stroop, especially because this was an untrained task. Although this study was not powered to detect cognitive gains, it showed the potential for motor-cognitive training in PD. These results reiterate the findings of a recent virtual reality training study ${ }^{28}$ and concur with recent review papers on cognitive training in PD. ${ }^{31-33}$ One of these reviews showed that executive function is most likely to improve not only after pure cognitive exercise but also after combined motor and cognitive training. ${ }^{31}$

In this study, the results on other DT cognitive outcomes were less clear. Reaction time on the DT digit span was longer, but fewer errors were made, suggesting a trade-off between task components. Training did not have an effect on manipulating the mobile phone during walking. However, a ceiling effect may have been present during DT cognitive performance. Effects on DT cognitive outcomes thus relied on the type of task and possibly on the priority assigned to each task. Indeed, people with a wide variety of neurological disorders demonstrated a similar task-specific effect of DT training with a tendency to favor gait parameters over cognition and balance. ${ }^{34}$ Hence, integrated DT training incorporating cognitive exercise may closely resemble ST cognitive training if patients prioritize this aspect, providing another explanation for the lack of difference between training modes.

Clinically, an important finding was the apparent safety of both interventions in contrast to the earlier notion that DT training would be fall-provoking in PD. ${ }^{10}$ We found that fall rates tended to drop during practice $(12 \%)$ and even more so in the first 6 weeks after training $(24 \%)$, but this was not statistically significant. This eye-catching result is even more notable as the sample included a considerable number of recurrent fallers $(33.1 \%)$ and may be explained by the fact that supervised training increased awareness of DT associated fall risk. The fact that fall frequency became worse when professional input was withdrawn supports this interpretation. Still, this study was not powered to analyze falls, constituting a limitation. The CTT group had a higher number of falls at baseline, which persisted throughout the study and showed no differential effect of training mode. It is possible that as the participants with more frequent falls received "the safest intervention" (CTT), true fall risk may have been masked.

Adherence rates in both arms were very good, with more than $80 \%$ of participants following more than $80 \%$ of sessions. ${ }^{35}$ As cognitive exercises are not part of standard physiotherapy care, it was encouraging to ascertain that participants were satisfied with this novel part of the intervention and tended to want to continue cognitive practice.

We powered the study based on a $15 \%$ difference of improvement between IDT and CTT. ${ }^{15}$ Yet we found only small differences between the 2 study arms, irrespective of dropout being larger than expected. This implies that clinicians can choose which strategy they wish to adopt to improve dual tasking in PD and individualize therapy according to clinical need and disease stage. The physiotherapists who provided the IDT and CTT training did so in both arms and this may have biased the results. However, the fact that most patients assumed that they had been in the most effective intervention argues against performance bias. Overall, we showed surprisingly well-preserved DT learning capacity regardless of clinical profile, even in patients with FOG $(56 \%)$ albeit that retention was impaired in this group. The relatively high number of 
people with FOG in this study may be explained by the fact that freezers were more aware of their DT problems and therefore may have been more ready to participate. It also enhances the generalizability of the findings, as it signifies that effect size estimates were probably conservative.

In conclusion, both CTT and IDT were found to be moderately and equally effective in improving DT gait velocity in people with $\mathrm{PD}$. This effect was apparent when compared with a control period without training and pertained to untrained cognitive and functional DT tasks. The fact that the effects were retained for 12 weeks illustrated a robust capacity for DT motor learning in PD. Contrary to current thinking, DT training proved not hazardous, as fall risk remained stable. These novel results support the European Physiotherapy Guideline for Parkinson's Disease, ${ }^{11}$ which recommends adopting DT training in clinical practice at least when supervised by rehabilitation professionals.

\section{Ethical Approval}

Full ethical approval has been granted for the study by The University Hospitals Leuven and the Radboud University Medical Centre Nijmegen (Belgium: CME KU Leuven - B322201213165/S53419; the Netherlands: CMO Regio Arnhem-Nijmegen - NL39530.091.12). All participants were asked to sign an informed consent form before study participation.

Acknowledgments: We would like to thank all patients with Parkinson's disease who participated in the study. We also thank all persons who assisted in study execution or gave statistical input.

\section{References}

1. Wu T, Hallett M. A functional MRI study of automatic movements in patients with Parkinson's disease. Brain 2005;128(10): 2250-2259.

2. Yarnall AJ, Breen DP, Duncan GW, et al. Characterizing mild cognitive impairment in incident Parkinson disease: the ICICLE-PD study. Neurology 2014;82(4):308-316.

3. Gratwicke J, Jahanshahi M, Foltynie T. Parkinson's disease dementia: a neural networks perspective. Brain 2015;138(6):1454-1476.

4. Bridenbaugh SA, Kressig RW. Motor cognitive dual tasking: early detection of gait impairment, fall risk and cognitive decline. Z Gerontol Geriatr 2015;48(1):15-21.

5. Kelly VE, Eusterbrock AJ, Shumway-Cook A. A review of dualtask walking deficits in people with Parkinson's disease: motor and cognitive contributions, mechanisms, and clinical implications. Parkinsons Dis 2012;2012:918719.

6. Rochester L, Galna B, Lord S, Burn D. The nature of dual-task interference during gait in incident Parkinson's disease. Neuroscience 2014;265:83-94.

7. Heinzel S, Maechtel M, Hasmann SE, et al. Motor dual-tasking deficits predict falls in Parkinson's disease: a prospective study. Parkinsonism Relat Disord 2016;26:73-77.

8. Spildooren J, Vercruysse S, Desloovere K, Vandenberghe W, Kerckhofs E, Nieuwboer A. Freezing of gait in Parkinson's disease: the impact of dual-tasking and turning. Mov Disord 2010;25(15): 2563-2570.

9. Fuller RL, Van Winkle EP, Anderson KE, et al. Dual task performance in Parkinson's disease: a sensitive predictor of impairment and disability. Parkinsonism Relat Disord 2013;19(3):325-328.
10. Keus SH, Bloem BR, Hendriks EJ, Bredero-Cohen AB, Munneke M, Practice Recommendations Development Group. Evidencebased analysis of physical therapy in Parkinson's disease with recommendations for practice and research. Mov Disord 2007;22(4): 451-460.

11. Keus SHJ, Munneke M, Graziano M, et al. European Physiotherapy Guideline for Parkinson's disease. The Netherlands: KNGF/ ParkinsonNet, Amersfoort/Nijmegen, 2014. www.parkinsonnet. info/euguideline

12. Strouwen C, Molenaar EA, Münks L, et al. Dual tasking in Parkinson's disease: should we train hazardous behavior? Expert Rev Neurother 2015;15(9):1031-1039.

13. Conradsson D, Lofgren N, Nero $\mathrm{H}$, et al. The effects of highly challenging balance training in elderly with Parkinson's disease: a randomized controlled trial. Neurorehabil Neural Repair 2015; 29(9):827-836.

14. Silsupadol P, Lugade V, Shumway-Cook A, et al. Training-related changes in dual-task walking performance of elderly persons with balance impairment: a double-blind, randomized controlled trial. Gait Posture 2009;29(4):634-639.

15. Strouwen C, Molenaar EA, Keus SH, et al. Protocol for a randomized comparison of integrated versus consecutive dual task practice in Parkinson's disease: the DUALITY trial. BMC Neurol 2014;14(1):61.

16. Hughes AJ, Daniel SE, Kilford L, Lees AJ. Accuracy of clinical diagnosis of idiopathic Parkinson's disease: a clinico-pathological study of 100 cases. J Neurol Neurosurg Psychiatry 1992;55(3):181-184.

17. Hoehn MM, Yahr MD. Parkinsonism: onset, progression and mortality. Neurology 1967;17(5):427-442.

18. Tombaugh TN, McIntyre NJ. The mini-mental state examination: a comprehensive review. J Am Geriatr Soc 1992;40(9):922-935.

19. Strouwen C, Molenaar EA, Keus SH, Münks L, Bloem BR, Nieuwboer A. Test-retest reliability of dual-task outcome measures in people with Parkinson's disease. Phys Ther 2016;96(8):1276-1286.

20. Menz HB, Latt MD, Tiedemann A, Mun San Kwan M, Lord SR. Reliability of the GAITRite ${ }^{\circledR}$ walkway system for the quantification of temporo-spatial parameters of gait in young and older people. Gait Posture 2004;20(1):20-25.

21. Ashburn A, Stack E, Ballinger C, Fazakarley L, Fitton C. The circumstances of falls among people with Parkinson's disease and the use of Falls Diaries to facilitate reporting. Disabil Rehabil 2008; 30(16):1205-1212.

22. Field A. Discovering Statistics Using IBM SPSS Statistics. 4th ed. London: SAGE 2013.

23. Deuschl G, Schade-Brittinger C, Krack P, et al. A randomized trial of deep-brain stimulation for Parkinson's disease. N Engl J Med 2006;355(9):896-908.

24. Liepelt R, Strobach T, Frensch P, Schubert T. Improved intertask coordination after extensive dual-task practice. Q J Exp Psychol (Hove) 2011;64(7):1251-1272.

25. Strobach T, Gerstorf D, Maquestiaux F, Schubert T. Does initial performance variability predict dual-task optimization with practice in younger and older adults? Exp Aging Res 2015;41(1):57-88.

26. Canning CG, Ada L, Woodhouse E. Multiple-task walking training in people with mild to moderate Parkinson's disease: a pilot study. Clin Rehabil 2008;22(3):226-233.

27. Brauer SG, Morris ME. Can people with Parkinson's disease improve dual tasking when walking? Gait Posture 2010;31(2): 229-233.

28. Mirelman A, Maidan I, Herman T, Deutsch JE, Giladi N, Hausdorff JM. Virtual reality for gait training: can it induce motor learning to enhance complex walking and reduce fall risk in patients with Parkinson's disease? J Gerontol A Biol Sci Med Sci 2011;66(2):234-240.

29. Perera S, Mody SH, Woodman RC, Studenski SA. Meaningful change and responsiveness in common physical performance measures in older adults. J Am Geriatr Soc 2006;54(5):743-749.

30. Yogev-Seligmann G, Giladi N, Brozgol M, Hausdorff JM. A training program to improve gait while dual tasking in patients with Parkinson's disease: a pilot study. Arch Phys Med Rehabil 2012; 93(1):176-181.

31. Hindle JV, Petrelli A, Clare L, Kalbe E. Nonpharmacological enhancement of cognitive function in Parkinson's disease: a systematic review. Mov Disord 2013;28(8):1034-1049. 
32. Leung IH, Walton CC, Hallock H, Lewis SJ, Valenzuela M, Lampit A. Cognitive training in Parkinson disease: a systematic review and meta-analysis. Neurology 2015;85(21):1843-1851.

33. David FJ, Robichaud JA, Leurgans SE, et al. Exercise improves cognition in Parkinson's disease: the PRET-PD randomized, clinical trial. Mov Disord 2015;30(12):1657-1663.

34. Fritz NE, Cheek FM, Nichols-Larsen DS. Motor-cognitive dualtask training in persons with neurologic disorders: a systematic review. J Neurol Phys Ther 2015;39(3):142-153.

35. Allen NE, Sherrington C, Suriyarachchi GD, Paul SS, Song J, Canning CG. Exercise and motor training in people with
Parkinson's disease: a systematic review of participant characteristics, intervention delivery, retention rates, adherence, and adverse events in clinical trials. Parkinsons Dis 2012;2012:854328.

\section{Supporting Data}

Additional Supporting Information may be found in the online version of this article at the publisher's web-site. 